\title{
Controllability results for impulsive differential systems with finite delay
}

\author{
S. Selvi ${ }^{a}$, M. Mallika Arjunan ${ }^{\mathrm{b}, *}$ \\ ${ }^{a}$ Department of Mathematics, Muthayammal College of Arts \& Science, Rasipuram- 637408, Tamil Nadu, India. \\ ${ }^{b}$ Department of Mathematics, Karunya University, Karunya Nagar, Coimbatore- 641 114, Tamil Nadu, India \\ This paper is dedicated to Professor Ljubomir Ćirić \\ Communicated by Professor V. Berinde
}

\begin{abstract}
This paper establishes some sufficient conditions for controllability of impulsive functional differential equations with finite delay in a Banach space. The results are obtained by using the measures of noncompactness and Monch fixed point theorem. Particularly, we do not assume the compactness of the evolution system. Finally, an example is provided to illustrate the theory.(C)2012 NGA. All rights reserved.
\end{abstract}

Keywords: Controllability, Impulsive differential equations, Measures of noncompactness, Semigroup theory, Fixed point.

2010 MSC: Primary 93B05, 34A37, 34G20.

\section{Introduction}

Impulsive differential equations have become more important in recent years in some mathematical models of real processes and phenomena studied in control, physics, chemistry, population dynamics, aeronautics and engineering. There has been a significant development in impulsive theory in recent years, especially in the area of impulsive differential equations with fixed moments, see the monographs of Bainov and Simeonov [3], Lakshmikantham et al. [14] and Samoilenko and Perestyuk [20] and the papers of [1, 2, 5, 7, 8, 9, 10, 12, 23]. On the other hand, differential equations with delay was initiated about existence and stability by Travis and Webb [21] and Webb 22]. Since such equations are often more realistic to describe natural phenomena than those without delay, they have been investigated in variant

\footnotetext{
* Corresponding author

Email addresses: sselvimaths@yahoo.com (S. Selvi), arjunphd07@yahoo.co.in (M. Mallika Arjunan)
} 
aspects by many authors [2, 15. The concept of controllability plays an important role in many areas of applied mathematics. In recent years, significant progress has been made in the controllability of linear and nonlinear deterministic systems [6, 11, 17, 19]. In [11, the author studied the controllability of impulsive functional differential systems of the form

$$
\begin{aligned}
x^{\prime}(t) & =A(t) x(t)+f(t, x(t))+(B u)(t), \text { a.e. on }[0, b], \\
\left.\Delta x\right|_{t=t_{i}} & =I_{i}\left(x\left(t_{i}\right)\right), i=1,2, \ldots, s, \\
x(0) & +M(x)=x_{0},
\end{aligned}
$$

where $A(t)$ is a family of linear operators which generates an evolution operator $U: \Delta=\{(t, s) \in[0, b] \times[0, b]$ : $0 \leq s \leq t \leq b\} \rightarrow L(X)$, here $X$ is a Banach space, $L(X)$ is the space of all bounded linear operators in $X ; f:[0, b] \times X \rightarrow X ; 0<t_{1}<\cdots<t_{s}<t_{s+1}=b ; I_{i}: X \rightarrow X, i=1,2, \ldots, s$, are impulsive functions; $M: P C([0, b], X) \rightarrow X ; B$ is a bounded linear operator from a Banach space $V$ to $X$ and the control function $u(\cdot)$ is given in $L^{2}([0, b], V)$. The results are obtained by using the measures of noncompactness and Monch fixed point theorem.

Motivated by the above mentioned works [7, 11, 15, 23], the main purpose of this paper is to establish the sufficient conditions for the controllability of impulsive differential system with finite delay of the form

$$
\begin{aligned}
x^{\prime}(t) & =A(t) x(t)+f\left(t, x_{t}\right)+(B u)(t), \\
t \in J & =[0, b], \quad t \neq t_{i}, i=1,2, \ldots, s, \\
\left.\Delta x\right|_{t=t_{i}} & =I_{i}\left(x_{t_{i}}\right), \quad i=1,2, \ldots, s, \\
x(t) & =\varphi(t), \quad t \in[-r, 0],
\end{aligned}
$$

where $A(t)$ is a family of linear operators which generates an evolution system $\{U(t, s): 0 \leq s \leq t \leq b\}$. The state variable $x(\cdot)$ takes the values in the real Banach space $X$ with norm $\|\cdot\|$. The control function $u(\cdot)$ is given in $L^{2}(J, V)$ a Banach space of admissible control functions with $V$ as a Banach space. $B$ is a bounded linear operator from $V$ into $X . f: J \times \mathcal{D} \rightarrow X$ is given function, where $\mathcal{D}=\{\psi:[-r, 0] \rightarrow X:$ $\psi(t)$ is continuous everywhere except for a finite number of points $t_{i}$ at which $\psi\left(t_{i}^{+}\right)$and $\psi\left(t_{i}^{-}\right)$exist and $\left.\psi\left(t_{i}\right)=\psi\left(t_{i}^{-}\right)\right\} ; I_{i}: \mathcal{D} \rightarrow X ; i=1,2, \ldots, s$, are impulsive functions, $0<t_{1}<t_{2}<\cdots<t_{s}<t_{s+1}=b$, $\Delta \xi\left(t_{i}\right)$ is the jump of a function $\xi$ at $t_{i}$, which is defined by $\Delta \xi\left(t_{i}\right)=\xi\left(t_{i}^{+}\right)-\xi\left(t_{i}^{-}\right)$.

For any function $x \in \mathcal{P C}$ and any $t \in J, x_{t}$ denotes the function in $\mathcal{D}$ defined by

$$
x_{t}(\theta)=x(t+\theta), \quad \theta \in[-r, 0] .
$$

where $\mathcal{P C}$ is defined in Preliminaries. Here $x_{t}(\cdot)$ represents the history of the state from the time $t-r$ upto the present time $t$. Our approach here is based on semigroup theory, measures of noncompactness and Monch fixed point theorem.

\section{Preliminaries}

In this section, we recall some basic definitions and lemmas which will be used to prove our main results of this paper.

Let $L^{1}([0, b], X)$ the space of $X$-valued Bochner integrable functions on $[0, \mathrm{~b}]$ with the norm $\|f\|_{L^{1}}=$ $\int_{0}^{b}\|f(t)\| d t$. In order to define the solution of the problem (1.1)-(1.3), we consider the following space:

$\mathcal{P C}([-r, b], X)=\left\{x:[-r, b] \rightarrow X\right.$ such that $x(\cdot)$ is continuous except for a finite number of points $t_{i}$ at which $x\left(t_{i}^{+}\right)$and $x\left(t_{i}^{-}\right)$exist and $\left.x\left(t_{i}\right)=x\left(t_{i}^{-}\right)\right\}$.

It is easy to verify that $\mathcal{P C}([-r, b], X)$ is a Banach space with the norm

$$
\|x\|_{\mathcal{P C}}=\sup \{\|x(t)\|: t \in[-r, b]\} .
$$

For our convenience let $\mathcal{P C}=\mathcal{P C}([-r, b], X)$ and $J_{0}=\left[0, t_{1}\right] ; \quad J_{i}=\left(t_{i}, t_{i+1}\right], i=1,2, \ldots, s$. 
Definition 2.1. Let $E^{+}$be the positive cone of an order Banach space $(E, \leq)$. A function $\Phi$ defined on the set of all bounded subsets of the Banach space $X$ with values in $E^{+}$is called a measure of noncompactness(MNC) on $X$ if $\Phi(\overline{c o} \Omega)=\Phi(\Omega)$ for all bounded subsets $\Omega \subseteq X$, where $\overline{c o} \Omega$ stands for the closed convex hull of $\Omega$.

The $M N C \Phi$ is said:

(1) Monotone if for all bounded subsets $\Omega_{1}, \Omega_{2}$ of $X$ we have: $\left(\Omega_{1} \subseteq \Omega_{2}\right) \Rightarrow\left(\Phi\left(\Omega_{1}\right) \leq \Phi\left(\Omega_{2}\right)\right)$;

(2) Nonsingular if $\Phi(\{a\} \cup \Omega)=\Phi(\Omega)$ for every $a \in X, \Omega \subset X$;

(3) Regular if $\Phi(\Omega)=0$ if and only if $\Omega$ is relatively compact in $X$.

One of the most examples of $M N C$ is the noncompactness measure of Hausdorff $\beta$ defined on each bounded subset $\Omega$ of $X$ by

$\beta(\Omega)=\inf \{\epsilon>0 ; \Omega$ can be covered by a finite number of balls of radii smaller than $\epsilon\}$

It is well known that MNC $\beta$ enjoys the above properties and other properties see [4, 13]: For all bounded subsets $\Omega, \Omega_{1}, \Omega_{2}$ of $X$,

(4) $\beta\left(\Omega_{1}+\Omega_{2}\right) \leq \beta\left(\Omega_{1}\right)+\beta\left(\Omega_{2}\right)$, where $\Omega_{1}+\Omega_{2}=\left\{x+y: x \in \Omega_{1}, y \in \Omega_{2}\right\}$;

(5) $\beta\left(\Omega_{1} \cup \Omega_{2}\right) \leq \max \left\{\beta\left(\Omega_{1}\right), \beta\left(\Omega_{2}\right)\right\}$;

(6) $\beta(\lambda \Omega) \leq|\lambda| \beta(\Omega)$ for any $\lambda \in \mathbb{R}$;

(7) If the map $Q: D(Q) \subseteq X \rightarrow Z$ is Lipschitz continuous with constant $k$, then $\beta_{Z}(Q \Omega) \leq k \beta(\Omega)$ for any bounded subset $\Omega \subseteq D(Q)$, where $Z$ is a Banach space.

Definition 2.2. A two parameter family of bounded linear operators $U(t, s), 0 \leq s \leq t \leq b$ on $X$ is called an evolution system if the following two conditions are satisfied:

(i) $U(s, s)=I, U(t, r) U(r, s)=U(t, s)$ for $0 \leq s \leq r \leq t \leq b$;

(ii) $(t, s) \rightarrow U(t, s)$ is strongly continuous for $0 \leq s \leq t \leq b$.

Since the evolution system $U(t, s)$ is strongly continuous on the compact operator set $J \times J$, then there exists $M_{1}>0$ such that $\|U(t, s)\| \leq M_{1}$ for any $(t, s) \in J \times J$. More details about evolution system can be found in Pazy [18].

Definition 2.3. A function $x(\cdot) \in \mathcal{P C}$ is said to be a mild solution of the system $(1.1)-(1.3)$ if, $x(t)=\varphi(t)$ on $[-r, 0] ;\left.\Delta x\right|_{t=t_{i}}=I_{i}\left(x_{t_{i}}\right), i=1,2 \ldots, s$; the restriction of $x(\cdot)$ to the interval $J_{i}(i=1,2, \ldots, s)$ is continuous and the following integral equation is satisfied.

$$
x(t)=U(t, 0) \varphi(0)+\int_{0}^{t} U(t, s)\left[B u(s)+f\left(s, x_{s}\right)\right] d s+\sum_{0<t_{i}<t} U\left(t, t_{i}\right) I_{i}\left(x_{t_{i}}\right), t \in J .
$$

Definition 2.4. The system (1.1) - (1.3) is said to be controllable on the interval $J$ if, for every initial function $\varphi \in \mathcal{D}$ and $x_{1} \in X$, there exists a control $u \in L^{2}(J, V)$ such that the mild solution $x(\cdot)$ of $(1.1)-(1.3)$ satisfies $x(b)=x_{1}$.

Definition 2.5. A countable set $\left\{f_{n}\right\}_{n=1}^{\infty} \subset L^{1}([0, b], X)$ is said to be semicompact if the sequence $\left\{f_{n}\right\}_{n=1}^{\infty}$ is relatively compact in $X$ for almost all $t \in[0, b]$ and if there is a function $\mu \in L^{1}\left([0, b], \mathbb{R}^{+}\right)$satisfying $\sup \left\|f_{n}(t)\right\| \leq \mu(t)$ for a.e. $t \in[0, b]$.

$n \geq 1$

Lemma 2.1. ([4]) If $W \subset C([a, b], X)$ is bounded and equicontinuous, then $\beta(W(t))$ is continuous for $t \in[a, b]$ and

$$
\beta(W)=\sup \{\beta(W(t)), t \in[a, b]\}, \quad \text { where } W(t)=\{x(t): x \in W\} \subseteq X .
$$


Lemma 2.2. ([23]) If $W \subset \mathcal{P C}([a, b], X)$ is bounded and piecewise equicontinuous on $[a, b]$ then $\beta(W(t))$ is piecewise continuous for $t \in[a, b]$ and

$$
\beta(W)=\sup \{\beta(W(t)), t \in[a, b]\} .
$$

Lemma 2.3. ([17]) Let $\left\{f_{n}\right\}_{n=1}^{\infty}$ be a sequence of functions in $L^{1}\left([0, b], \mathbb{R}^{+}\right)$. Assume that there exist $\mu, \eta \in L^{1}\left([0, b], \mathbb{R}^{+}\right)$satisfying $\sup _{n \geq 1}\left\|f_{n}(t)\right\| \leq \mu(t)$ and $\beta\left(\left\{f_{n}(t)\right\}_{n=1}^{\infty}\right) \leq \eta(t)$ a.e. $t \in[0, b]$, then for all $t \in[0, b]$, we have

$$
\beta\left(\left\{\int_{0}^{t} U(t, s) f_{n}(s) d s: n \geq 1\right\}\right) \leq 2 M_{1} \int_{0}^{t} \eta(s) d s .
$$

Lemma 2.4. ([17]) Let $(G f)(t)=\int_{0}^{t} U(t, s) f(s) d s$, If $\left\{f_{n}\right\}_{n=1}^{\infty} \subset L^{1}([0, b], X)$ is semicompact, then the set $\left\{G f_{n}\right\}_{n=1}^{\infty}$ is relatively compact in $C([0, b], X)$ and moreover if $f_{n} \rightarrow f_{0}$, then for all $t \in[0, b]$,

$$
\left(G f_{n}\right)(t) \rightarrow\left(G f_{0}\right)(t) \text {, as } n \rightarrow \infty .
$$

The following fixed-point theorem, a nonlinear alternative of Monch type, plays a key role in our proof of controllability of the system (1.1) - (1.3).

Lemma 2.5. ([16, Theorem 2.2]) Let $D$ be a closed convex subset of a Banach space $X$ and $0 \in D$. Assume that $F: D \rightarrow X$ is a continuous map which satisfies Monch's condition, that is $(M \subseteq D$ is countable, $M \subseteq \overline{c o}(\{0\} \cup F(M)) \Rightarrow \bar{M}$ is compact $)$. Then $F$ has a fixed point in $D$.

\section{Controllability Results}

In this section, we present and prove the controllability results for the problem $(1.1)-(1.3)$. In order to prove the main theorem of this section, we list the following hypotheses:

(H1) $A(t)$ is a family of linear operators, $A(t): D(A) \rightarrow X, D(A)$ not depending on $\mathrm{t}$ and dense subset of $X$, generating an equicontinuous evolution system $\{U(t, s): 0 \leq s \leq t \leq b\}$, i.e., $(t, s) \rightarrow\{U(t, s) x: x \in$ $B\}$ is equicontinuous for $t>0$ and for all bounded subsets $B$ and $M_{1}=\sup \{\|U(t, s)\|:(t, s) \in J \times J\}$.

(H2) The function $f: J \times \mathcal{D} \rightarrow X$ satisfies:

(i) For a.e. $t \in J$, the function $f(t, \cdot): \mathcal{D} \rightarrow X$ is continuous and for all $\varphi \in \mathcal{D}$, the function $f(\cdot, \varphi): J \rightarrow X$ is strongly measurable.

(ii) For every positive integer $r$, there exists $\alpha_{r} \in L^{1}\left([0, b] ; \mathbb{R}^{+}\right)$such that

$$
\sup _{\|\varphi\|_{\mathcal{D}} \leq r}\|f(t, \varphi)\| \leq \alpha_{r}(t) \text { for a.e. } t \in J
$$

and

$$
\lim _{r \rightarrow \infty} \inf \int_{0}^{b} \frac{\alpha_{r}(t)}{r} d t=\sigma<\infty
$$

(iii) There exists integrable function $\eta:[0, b] \rightarrow[0, \infty)$ such that

$$
\beta(f(t, D)) \leq \eta(t) \sup _{-r \leq \theta \leq 0} \beta(D(\theta)) \quad \text { for a.e. } t \in J \text { and } D \subset \mathcal{D},
$$

where $D(\theta)=\{v(\theta): v \in D\}$.

(H3) The linear operator $W: L^{2}(J, V) \rightarrow X$ is defined by

$$
W u=\int_{0}^{b} U(t, s) B u(s) d s \quad \text { such that }
$$


(i) $W$ has an invertible operator $W^{-1}$ which take values in $L^{2}(J, V) \backslash \operatorname{ker} W$, and there exist positive constants $M_{2}$ and $M_{3}$ such that

$$
\|B\| \leq M_{2},\left\|W^{-1}\right\| \leq M_{3} .
$$

(ii) There is $K_{W} \in L^{1}\left(J, \mathbb{R}^{+}\right)$such that, for every bounded set $Q \subset X$,

$$
\beta\left(W^{-1} Q\right)(t) \leq K_{W}(t) \beta(Q) .
$$

(H4) $I_{i}: \mathcal{D} \rightarrow X, i=1,2 \ldots, s$, be a continuous operator such that:

(i) There are nondecreasing functions $L_{i}: \mathbb{R}^{+} \rightarrow \mathbb{R}^{+}$such that

$$
\left\|I_{i}(x)\right\| \leq L_{i}\left(\|x\|_{\mathcal{D}}\right) \quad i=1,2 \ldots, s, \quad x \in \mathcal{D},
$$

and

$$
\lim _{\rho \rightarrow \infty} \inf \frac{L_{i}(\rho)}{\rho}=\lambda_{i}<\infty, \quad i=1,2 \ldots, s .
$$

(ii) There exist constants $K_{i} \geq 0$ such that,

$$
\beta\left(I_{i}(S)\right) \leq K_{i} \sup _{-r \leq \theta \leq 0} \beta(S(\theta)), \quad i=1,2 \ldots, s,
$$

for every bounded subset $S$ of $\mathcal{D}$.

(H5) The following estimation holds true:

$$
N=\left[\left(M_{1}+2 M_{1}^{2} M_{2}\left\|K_{W}\right\|_{L^{1}}\right) \sum_{i=1}^{s} K_{i}+\left(2 M_{1}+4 M_{1}^{2} M_{2}\left\|K_{W}\right\|_{L^{1}}\right)\|\eta\|_{L^{1}}\right]<1 .
$$

Theorem 3.1. Assume that the hypotheses $(H 1)-(H 5)$ are satisfied. Then the impulsive differential system $(1.1)-(1.3)$ is controllable on $J$ provided that,

$$
M_{1}\left(1+M_{1} M_{2} M_{3} b^{\frac{1}{2}}\right)\left(\sigma+\sum_{i=1}^{s} \lambda_{i}\right)<1 .
$$

Proof. Using the hypothesis $(H 3)(i)$, for every $x \in \mathcal{P C}([-r, b], X)$, define the control

$$
u_{x}(t)=W^{-1}\left[x_{1}-U(b, 0) \varphi(0)-\int_{0}^{b} U(b, s) f\left(s, x_{s}\right) d s-\sum_{0<t_{i}<b} U\left(b, t_{i}\right) I_{i}\left(x_{t_{i}}\right)\right](t) .
$$

We shall now show that when using this control the operator defined by

$$
(F x)(t)=\left\{\begin{array}{l}
\varphi(t), \quad t \in[-r, 0], \\
U(t, 0) \varphi(0)+\int_{0}^{t} U(t, s)\left[f\left(s, x_{s}\right)+\left(B u_{x}\right)(s)\right] d s \\
\quad+\sum_{0<t_{i}<t} U\left(t, t_{i}\right) I_{i}\left(x_{t_{i}}\right), \quad t \in J,
\end{array}\right.
$$

has a fixed point. This fixed point is then a solution of $(1.1)-(1.3)$. Clearly $x(b)=(F x)(b)=x_{1}$, which implies the system $(1.1)-(1.3)$ is controllable. We rewrite the problem (1.1) - (1.3) as follows:

For $\varphi \in \mathcal{D}$, we define $\hat{\varphi} \in \mathcal{P C}$ by

$$
\hat{\varphi}(t)=\left\{\begin{array}{l}
U(t, 0) \varphi(0), \quad t \in J \\
\varphi(t), \quad t \in[-r, 0]
\end{array}\right.
$$


Then $\hat{\varphi} \in \mathcal{P C}$. Let $x(t)=y(t)+\hat{\varphi}(t), t \in[-r, b]$. It is easy to see that $y$ satisfies $y_{0}=0$ and

$$
y(t)=\int_{0}^{t} U(t, s)\left[f\left(s, y_{s}+\hat{\varphi}_{s}\right)+B u_{y}(s)\right] d s+\sum_{0<t_{i}<t} U\left(t, t_{i}\right) I_{i}\left(y_{t_{i}}+\hat{\varphi}_{t_{i}}\right),
$$

where

$$
\begin{aligned}
u_{y}(s)= & W^{-1}\left[x_{1}-U(b, 0) \varphi(0)-\int_{0}^{b} U(b, s) f\left(s, y_{s}+\hat{\varphi}_{s}\right) d s\right. \\
& \left.-\sum_{i=1}^{s} U\left(b, t_{i}\right) I_{i}\left(y_{t_{i}}+\hat{\varphi}_{t_{i}}\right)\right](s),
\end{aligned}
$$

if and only if $x$ satisfies

$$
x(t)=U(t, 0) \varphi(0)+\int_{0}^{t} U(t, s)\left[f\left(s, x_{s}\right)+B u_{x}(s)\right] d s+\sum_{0<t_{i}<t} U\left(t, t_{i}\right) I_{i}\left(x_{t_{i}}\right),
$$

and $x(t)=\varphi(t), t \in[-r, 0]$. Define $\mathcal{P C} \mathcal{C}_{0}=\left\{y \in \mathcal{P C}: y_{0}=0\right\}$. Let $G: \mathcal{P C}_{0} \rightarrow \mathcal{P C}_{0}$ be an operator defined by

$$
(G y)(t)=\left\{\begin{array}{l}
0, \quad t \in[-r, 0], \\
\int_{0}^{t} U(t, s)\left[f\left(s, y_{s}+\hat{\varphi}_{s}\right)+B u_{y}(s)\right] d s \\
\quad+\sum_{0<t_{i}<t} U\left(t, t_{i}\right) I_{i}\left(y_{t_{i}}+\hat{\varphi}_{t_{i}}\right), \quad t \in J .
\end{array}\right.
$$

Obviously the operator $F$ has a fixed point is equivalent to $G$ has one. So it turns out to prove $G$ has a fixed point.

Let $G=G_{1}+G_{2}$,

where

$$
\begin{aligned}
& \left(G_{1} y\right)(t)=\sum_{0<t_{i}<t} U\left(t, t_{i}\right) I_{i}\left(y_{t_{i}}+\hat{\varphi}_{t_{i}}\right), \\
& \left(G_{2} y\right)(t)=\int_{0}^{t} U(t, s)\left[f\left(s, y_{s}+\hat{\varphi}_{s}\right)+B u_{y}(s)\right] d s .
\end{aligned}
$$

Step 1: There exists a positive number $q \geq 1$ such that $G\left(B_{q}\right) \subseteq B_{q}$, where $B_{q}=\left\{y \in \mathcal{P C} \mathcal{C}_{0}:\|y\|_{\mathcal{P C}} \leq q\right\}$. Suppose the contrary. Then for each positive integer $q$, there exists a function $y^{q}(\cdot) \in B_{q}$ but $G\left(y^{q}\right) \notin B_{q}$. i.e., $\left\|G\left(y^{q}\right)(t)\right\|>q$ for some $t \in J$.

We have from $(H 1)-(H 4)$,

$$
\begin{aligned}
q & <\left\|\left(G y^{q}\right)(t)\right\| \\
& \leq M_{1} \int_{0}^{b}\left\|f\left(s, y_{s}^{q}+\hat{\varphi}_{s}\right)+B u_{y^{q}}(s)\right\| d s+M_{1} \sum_{i=1}^{s} L_{i}\left(\left\|y_{t_{i}}^{q}+\hat{\varphi}_{t_{i}}\right\|_{\mathcal{D}}\right) \\
& \leq M_{1} \int_{0}^{b} \alpha_{q^{\prime}}(s) d s+M_{1} \int_{0}^{b}\left\|B u_{y^{q}}(s)\right\| d s+M_{1} \sum_{i=1}^{s} L_{i}\left(q^{\prime}\right) \\
& \leq M_{1} \int_{0}^{b} \alpha_{q^{\prime}}(s) d s+M_{1} M_{2} b^{\frac{1}{2}}\left\|u_{y^{q}}\right\|_{L^{2}}+M_{1} \sum_{i=1}^{s} L_{i}\left(q^{\prime}\right),
\end{aligned}
$$


where

$$
\left\|u_{y^{q}}\right\|_{L^{2}} \leq M_{3}\left[\left\|x_{1}\right\|+M_{1}\|\varphi\|_{\mathcal{D}}+M_{1} \int_{0}^{b} \alpha_{q^{\prime}}(s) d s+M_{1} \sum_{i=1}^{s} L_{i}\left(q^{\prime}\right)\right] .
$$

Hence by (3.5),

$$
\begin{gathered}
q<M_{1} \int_{0}^{b} \alpha_{q^{\prime}}(s) d s+M_{1} M_{2} b^{\frac{1}{2}} M_{3}\left[\left\|x_{1}\right\|+M_{1}\|\varphi\|_{\mathcal{D}}+M_{1} \int_{0}^{b} \alpha_{q^{\prime}}(s) d s\right. \\
\left.\quad+M_{1} \sum_{i=1}^{s} L_{i}\left(q^{\prime}\right)\right]+M_{1} \sum_{i=1}^{s} L_{i}\left(q^{\prime}\right) \\
\leq\left(1+M_{1} M_{2} M_{3} b^{\frac{1}{2}}\right) M_{1}\left[\int_{0}^{b} \alpha_{q^{\prime}}(s) d s+\sum_{i=1}^{s} L_{i}\left(q^{\prime}\right)\right]+M,
\end{gathered}
$$

where $M=M_{1} M_{2} M_{3} b^{\frac{1}{2}}\left(\left\|x_{1}\right\|+M_{1}\|\varphi\|_{\mathcal{D}}\right)$ is independent of $q$ and $q^{\prime}=q+\|\hat{\varphi}\|_{\mathcal{P C}}$.

Dividing both sides by $q$ and noting that $q^{\prime}=q+\|\hat{\varphi}\|_{\mathcal{P C}} \rightarrow \infty$ as $q \rightarrow \infty$. We obtain

$$
\begin{array}{r}
\lim _{q \rightarrow+\infty} \inf \left(\frac{\int_{0}^{b} \alpha_{q^{\prime}}(s) d s}{q}\right)=\lim _{q \rightarrow+\infty} \inf \left(\frac{\int_{0}^{b} \alpha_{q^{\prime}}(s) d s}{q^{\prime}} \cdot \frac{q^{\prime}}{q}\right)=\sigma, \\
\lim _{q \rightarrow+\infty} \inf \left(\frac{\sum_{i=1}^{s} L_{i}\left(q^{\prime}\right)}{q}\right)=\lim _{q \rightarrow+\infty} \inf \left(\frac{\sum_{i=1}^{s} L_{i}\left(q^{\prime}\right)}{q^{\prime}} \cdot \frac{q^{\prime}}{q}\right)=\sum_{i=1}^{s} \lambda_{i} .
\end{array}
$$

Thus we have

$$
1 \leq M_{1}\left(1+M_{1} M_{2} M_{3} b^{\frac{1}{2}}\right)\left(\sigma+\sum_{i=1}^{s} \lambda_{i}\right) .
$$

This contradicts (3.1). Hence for some positive number $q, G\left(B_{q}\right) \subseteq B_{q}$.

Step 2: $G: \mathcal{P C}_{0} \rightarrow \mathcal{P C} \mathcal{C}_{0}$ is continuous.

Let $\left\{y^{(n)}(t)\right\}_{n=1}^{\infty} \subseteq \mathcal{P} \mathcal{C}_{0}$ with $y^{(n)} \rightarrow y$ in $\mathcal{P C}_{0}$. Then there is a number $q>0$ such that $\left\|y^{(n)}(t)\right\| \leq q$ for all $n$ and a.e. $t \in J$, so $y^{(n)} \in B_{q}$ and $y \in B_{q}$. By $\left(H_{2}\right)(i), f\left(t, y_{t}^{(n)}+\hat{\varphi}_{t}\right) \rightarrow f\left(t, y_{t}+\hat{\varphi}_{t}\right)$ for each $t \in J$. By $\left(H_{2}\right)(i i),\left\|f\left(t, y_{t}^{(n)}+\hat{\varphi}_{t}\right)-f\left(t, y_{t}+\hat{\varphi}_{t}\right)\right\|<2 \alpha_{q^{\prime}}(t)$ and by $\left(H_{4}\right), I_{i}\left(y_{t_{i}}^{(n)}+\hat{\varphi}_{t_{i}}\right) \rightarrow I_{i}\left(y_{t_{i}}+\hat{\varphi}_{t_{i}}\right), i=1,2, \ldots, s$. Then we have

$$
\left\|G_{1} y^{(n)}-G_{1} y\right\|_{\mathcal{P C}} \leq M_{1} \sum_{i=1}^{s}\left\|I_{i}\left(y_{t_{i}}^{(n)}+\hat{\varphi}_{t_{i}}\right)-I_{i}\left(y_{t_{i}}+\hat{\varphi}_{t_{i}}\right)\right\| .
$$

and

$$
\begin{aligned}
& \left\|G_{2} y^{(n)}-G_{2} y\right\|_{\mathcal{P C}} \\
& \leq M_{1} \int_{0}^{b}\left\|f\left(s, y_{s}^{(n)}+\hat{\varphi}_{s}\right)-f\left(s, y_{s}+\hat{\varphi}_{s}\right)\right\| d s+M_{1} M_{2} \int_{0}^{b}\left\|u_{y^{(n)}}(s)-u_{y}(s)\right\| d s \\
& \leq M_{1} \int_{0}^{b}\left\|f\left(s, y_{s}^{(n)}+\hat{\varphi}_{s}\right)-f\left(s, y_{s}+\hat{\varphi}_{s}\right)\right\| d s+M_{1} M_{2} b^{\frac{1}{2}}\left\|u_{y}^{(n)}-u_{y}\right\|_{L^{2}},
\end{aligned}
$$

where

$$
\begin{aligned}
\left\|u_{y}^{(n)}-u_{y}\right\|_{L^{2}} \leq & M_{3}\left[M_{1} \int_{0}^{b}\left\|f\left(s, y_{s}^{(n)}+\hat{\varphi}_{s}\right)-f\left(s, y_{s}+\hat{\varphi}_{s}\right)\right\| d s\right. \\
& \left.+M_{1} \sum_{i=1}^{s}\left\|I_{i}\left(y_{t_{i}}^{(n)}+\hat{\varphi}_{t_{i}}\right)-I_{i}\left(y_{t_{i}}+\hat{\varphi}_{t_{i}}\right)\right\|\right]
\end{aligned}
$$


Observing (3.7) - (3.9) and by dominated convergence theorem we have that,

$$
\left\|G y^{(n)}-G y\right\|_{\mathcal{P C}} \leq\left\|G_{1} y^{(n)}-G y\right\|_{\mathcal{P C}}+\left\|G_{2} y^{(n)}-G_{2} y\right\|_{\mathcal{P C}} \rightarrow 0, \quad \text { as } n \rightarrow+\infty .
$$

That is $G$ is continuous.

Step 3: $G\left(B_{q}\right)$ is equicontinuous on every $J_{i}, i=1,2, \ldots, s$. That is $G\left(B_{q}\right)$ is piecewise equicontinuous on $J$.

Indeed for $t_{1}, t_{2} \in J_{i}, t_{1}<t_{2}$ and $y \in B_{q}$, we deduce that

$$
\begin{aligned}
& \left\|(G y)\left(t_{2}\right)-(G y)\left(t_{1}\right)\right\| \\
& \leq \int_{0}^{t_{1}}\left\|U\left(t_{2}, s\right)-U\left(t_{1}, s\right)\right\|\left\|f\left(s, y_{s}+\hat{\varphi}_{s}\right)+B u_{y}(s)\right\| d s \\
& \quad+\int_{t_{1}}^{t_{2}}\left\|U\left(t_{2}, s\right)\right\| f\left(s, y_{s}+\hat{\varphi}_{s}\right)+B u_{y}(s) \| d s \\
& \leq \int_{0}^{t_{1}}\left\|U\left(t_{2}, s\right)-U\left(t_{1}, s\right)\right\| \alpha_{q^{\prime}}(s) d s+\int_{0}^{t_{1}}\left\|U\left(t_{2}, s\right)-U\left(t_{1}, s\right)\right\| M_{2} M_{3}\left[\left\|x_{1}\right\|\right. \\
& \left.\quad+M_{1}\|\varphi(0)\|+M_{1} \int_{0}^{b} \alpha_{q^{\prime}} d s+M_{1} \sum_{i=1}^{t_{2}} L_{i}\left(q^{\prime}\right)\right] d s+\int_{t_{1}}^{t_{2}}\left\|U\left(t_{2}, s\right)\right\| \alpha_{q^{\prime}}(s) d s \\
& \quad+\int_{t_{1}}^{t_{2}}\left\|U\left(t_{2}, s\right)\right\| M_{2} M_{3}\left[\left\|x_{1}\right\|+M_{1}\|\varphi(0)\|+M_{1} \int_{0}^{b} \alpha_{q^{\prime}} d s+M_{1} \sum_{i=1}^{s} L_{i}\left(q^{\prime}\right)\right] d s .
\end{aligned}
$$

By the equicontinuity of $U(\cdot, s)$ and the absolute continuity of the Lebesgue integral, we can see that the right hand side of (3.10) tends to zero and independent of $y$ as $t_{2} \rightarrow t_{1}$. Hence $G\left(B_{q}\right)$ is equicontinuous on $J_{i}(i=1,2, \ldots, s)$.

Step 4: The Monch's condition holds.

Suppose $W \subseteq B_{q}$ is countable and $W \subseteq \overline{c o}(\{0\} \cup G(W))$. We shall show that $\beta(W)=0$, where $\beta$ is the Hausdorff MNC.

Without loss of generality, we may assume that $W=\left\{y^{(n)}\right\}_{n=1}^{\infty}$. Since $G$ maps $B_{q}$ into an equicontinuous family, $G(W)$ is equicontinuous on $J_{i}$. Hence $W \subseteq \overline{c o}(\{0\} \cup G(W))$ is also equicontinuous on every $J_{i}$.

By $\left(H_{4}\right)(i i)$, we have

$$
\begin{aligned}
& \beta\left(\left\{G_{1} y^{(n)}(t)\right\}_{n=1}^{\infty}\right) \\
= & \beta\left(\left\{\sum_{0<t_{i}<t} U\left(t, t_{i}\right) I_{i}\left(y_{t_{i}}^{(n)}+\hat{\varphi}_{t_{i}}\right)\right\}_{n=1}^{\infty}\right) \\
\leq & M_{1} \sum_{i=1}^{s} \beta\left(\left\{I_{i}\left(y_{t_{i}}^{(n)}+\hat{\varphi}_{t_{i}}\right)\right\}_{n=1}^{\infty}\right) \\
\leq & M_{1} \sum_{i=1}^{s} K_{i} \sup _{-r \leq \theta \leq 0} \beta\left(\left\{y^{(n)}\left(t_{i}+\theta\right)+\hat{\varphi}\left(t_{i}+\theta\right)\right\}_{n=1}^{\infty}\right) \\
\leq & M_{1} \sum_{i=1}^{s} K_{i} \sup _{0 \leq \tau_{i} \leq t_{i}} \beta\left(\left\{y^{(n)}\left(\tau_{i}\right)\right\}_{n=1}^{\infty}\right) .
\end{aligned}
$$


By Lemma 2.3 and from $(H 2)(i i i),(H 3)(i i)$ and $(H 4)(i i)$, we have that

$$
\begin{aligned}
\beta_{V}\left(\left\{u_{y^{(n)}}(s)\right\}_{n=1}^{\infty}\right) \leq & K_{W}(s)\left[\beta\left(\left\{\int_{0}^{b} U(b, s) f\left(s, y_{s}^{(n)}+\hat{\varphi}_{s}\right) d s\right\}_{n=1}^{\infty}\right)\right. \\
+ & \left.\left.\beta\left(\left\{\sum_{i=1}^{s} U\left(b, t_{i}\right) I_{i}\left(y_{t_{i}}^{(n)}+\hat{\varphi}_{t_{i}}\right)\right)\right\}_{n=1}^{\infty}\right)\right] \\
\leq & K_{W}(s)\left[2 M_{1} \int_{0}^{b} \eta(s) \sup _{-r \leq \theta \leq 0} \beta\left(\left\{y^{(n)}(s+\theta)+\hat{\varphi}(s+\theta)\right\}_{n=1}^{\infty}\right) d s\right. \\
& \left.+M_{1} \sum_{i=1}^{s} K_{i} \sup _{-r \leq \theta \leq 0} \beta\left(\left\{y^{(n)}\left(t_{i}+\theta\right)+\hat{\varphi}\left(t_{i}+\theta\right)\right\}_{n=1}^{\infty}\right)\right] \\
\leq & K_{W}(s)\left[2 M_{1} \int_{0}^{b} \eta(s) \sup _{0 \leq \tau \leq s} \beta\left(\left\{y^{(n)}(\tau)\right\}_{n=1}^{\infty}\right) d s\right. \\
& \left.+M_{1} \sum_{i=1}^{s} K_{i} \sup _{0 \leq \tau_{i} \leq t_{i}} \beta\left(\left\{y^{(n)}\left(\tau_{i}\right)\right\}_{n=1}^{\infty}\right)\right] .
\end{aligned}
$$

This implies that

$$
\begin{aligned}
\beta( & \left.\left\{G_{2} y^{(n)}(t)\right\}_{n=1}^{\infty}\right) \\
\leq & \beta\left(\left\{\int_{0}^{t} U(t, s) f\left(s, y_{s}^{(n)}+\hat{\varphi}_{s}\right) d s\right\}_{n=1}^{\infty}\right)+\beta\left(\left\{\int_{0}^{t} U(t, s) B u_{y^{(n)}}(s) d s\right\}_{n=1}^{\infty}\right) \\
\leq & 2 M_{1} \int_{0}^{b} \eta(s) \sup _{-r \leq \theta \leq 0} \beta\left(\left\{y^{(n)}(s+\theta)+\hat{\varphi}(s+\theta)\right\}_{n=1}^{\infty}\right) d s \\
& +2 M_{1} M_{2} \int_{0}^{b} \beta_{V}\left(\left\{u_{y^{(n)}}(s)\right\}_{n=1}^{\infty}\right) d s \\
\leq & 2 M_{1} \int_{0}^{b} \eta(s) \sup _{0 \leq \tau \leq s} \beta\left(\left\{y^{(n)}(\tau)\right\}_{n=1}^{\infty}\right) d s+4 M_{1}^{2} M_{2}\left(\int_{0}^{b} K_{W}(s) d s\right) \\
& \times\left(\int_{0}^{b} \eta(s) \sup _{0 \leq \tau \leq s} \beta\left(\left\{y^{(n)}(\tau)\right\}_{n=1}^{\infty}\right) d s\right) \\
& +2 M_{1}^{2} M_{2} \int_{0}^{b} K_{W}(s) d s\left(\sum_{i=1}^{s} K_{i} \sup _{0 \leq \tau_{i} \leq t_{i}} \beta\left(\left\{y^{(n)}\left(\tau_{i}\right)\right\}_{n=1}^{\infty}\right)\right.
\end{aligned}
$$

for each $t \in J$. From (3.11) and (3.13) we obtain that

$$
\begin{aligned}
& \beta\left(\left\{G y^{(n)}(t)\right\}_{n=1}^{\infty}\right) \\
& \leq \beta\left(\left\{G_{1} y^{(n)}(t)\right\}_{n=1}^{\infty}\right)+\beta\left(\left\{G_{2} y^{(n)}(t)\right\}_{n=1}^{\infty}\right) \\
& \leq M_{1} \sum_{i=1}^{s} K_{i} \sup _{0 \leq \tau_{i} \leq t_{i}} \beta\left(\left\{y^{(n)}\left(\tau_{i}\right)\right\}_{n=1}^{\infty}\right)+\left(2 M_{1}+4 M_{1}^{2} M_{2} \int_{0}^{b} K_{W}(s) d s\right) \\
& \quad \times \int_{0}^{b} \eta(s) \sup _{0 \leq \tau \leq s} \beta\left(\left\{y^{(n)}(\tau)\right\}_{n=1}^{\infty}\right) d s \\
& \quad+2 M_{1}^{2} M_{2} \int_{0}^{b} K_{W}(s) d s\left(\sum_{i=1}^{s} K_{i} \sup _{0 \leq \tau_{i} \leq t_{i}} \beta\left(\left\{y^{(n)}\left(\tau_{i}\right)\right\}_{n=1}^{\infty}\right)\right)
\end{aligned}
$$

for each $t \in J$. 
Since $W$ and $G(W)$ are equicontinuous on every $J_{i}$, according to Lemma 2.2, the inequality (3.14) implies that,

$$
\begin{aligned}
\beta( & \left.\left\{G y^{(n)}\right\}_{n=1}^{\infty}\right) \\
\leq & {\left[M_{1} \sum_{i=1}^{s} K_{i}+\left(2 M_{1}+4 M_{1}^{2} M_{2}\left\|K_{W}\right\|_{L^{1}}\right)\|\eta\|_{L^{1}}\right] \beta\left(\left\{y^{(n)}\right\}_{n=1}^{\infty}\right) } \\
& +\left[2 M_{1}^{2} M_{2}\left\|K_{W}\right\|_{L^{1}} \sum_{i=1}^{s} K_{i}\right] \beta\left(\left\{y^{(n)}\right\}_{n=1}^{\infty}\right) \\
= & {\left[\left(M_{1}+2 M_{1}^{2} M_{2}\left\|K_{W}\right\|_{L^{1}}\right) \sum_{i=1}^{s} K_{i}+\left(2 M_{1}+4 M_{1}^{2} M_{2}\left\|K_{W}\right\|_{L^{1}}\right)\|\eta\|_{L^{1}}\right] \beta\left(\left\{y^{(n)}\right\}_{n=1}^{\infty}\right) } \\
= & N \beta\left(\left\{y^{(n)}\right\}_{n=1}^{\infty}\right) .
\end{aligned}
$$

That is $\beta(G W) \leq N \beta(W)$, where $N$ is defined in (H5). Thus from the Monch's condition, we get that

$$
\beta(W) \leq \beta(\overline{c o}(\{0\} \cup G(W))=\beta(G(W)) \leq N \beta(W),
$$

since $N<1$, which implies that $\beta(W)=0$. So we have that $W$ is relatively compact in $\mathcal{P} \mathcal{C}_{0}$. In the view of Lemma 2.5, i.e., Monch's fixed point theorem, we conclude that $G$ has a fixed point $y$ in $W$. Then $x=y+\hat{\varphi}$ is a fixed point of $F$ in $\mathcal{P C}$ and thus the system $(1.1)-(1.3)$ is controllable on $[0, b]$.

Remark 3.1. Note that if $f$ is compact or Lipschitz continuous, then (H2)(iii) is automatically satisfied. In the following, by using another $M N C$, we will prove the result of the Theorem 3.1 in the case there is no equicontinuity of the evolution system $U(t, s)$ and hypothesis $(H 5)$. Here we assume that the impulsive operators $I_{i}$ are compact. So, instead of $(H 4)$, we give the hypothesis $(H 4)^{\prime}$ :

$\left(H 4^{\prime}\right) I_{i}: \mathcal{D} \rightarrow X, i=1,2 \ldots, s$, be a continuous compact operator such that, there are nondecreasing functions $L_{i}: \mathbb{R}^{+} \rightarrow \mathbb{R}^{+}$satisfying

$$
\left\|I_{i}(x)\right\| \leq L_{i}\left(\|x\|_{\mathcal{D}}\right) \quad i=1,2 \ldots, s, \quad x \in \mathcal{D},
$$

and

$$
\lim _{\rho \rightarrow \infty} \inf \frac{L_{i}(\rho)}{\rho}=\lambda_{i}<\infty, \quad i=1,2 \ldots, s .
$$

Theorem 3.2. Let $\{A(t)\}_{t \in[0, b]}$ be a family of linear operators that generates a strongly continuous evolution system $\{U(t, s):(t, s) \in J \times J\}$. Assume that the hypothesis $(H 2),(H 3)$ and $\left(H 4^{\prime}\right)$ are satisfied. Then the impulsive differential system $(1.1)-(1.3)$ is controllable on $J$.

Proof. In the view of Theorem 3.1, we should only prove that the function $G: \mathcal{P C}_{0} \rightarrow \mathcal{P C}$ given by the formula (3.2) satisfies the Monch's condition.

For this purpose, let $W \subseteq B_{q}$ be countable and $W \subseteq \overline{c o}(\{0\} \cup G(W))$. We shall prove that $W$ is relatively compact.

We will denote by $\Phi$ the following $\mathrm{MNC}$ in $\mathcal{P C}_{0}$ defined by (see[13]),

$$
\Phi(\Omega)=\max _{E \in \Delta(\Omega)}\left(\alpha(E), \bmod _{c}(E)\right) .
$$

for all bounded subsets of $\Omega$ of $\mathcal{P C}_{0}$, where $\Delta(\Omega)$ is the set of countable subsets of $\Omega, \alpha$ is the real MNC defined by,

$$
\alpha(E)=\sup _{t \in[0, b]} e^{-L t} \beta(E(t)),
$$


with $E(t)=\{x(t): x \in E\}, \mathrm{L}$ is a constant that we shall choose appropriately.

$\bmod { }_{c}(E)$ is the modulus of equicontinuity of the function set $E$ given by the formula

$$
\bmod { }_{c}(E)=\lim _{\delta \rightarrow 0} \sup _{x \in E} \max _{0 \leq i \leq s} \max _{t_{1}, t_{2} \in J_{i},\left\|t_{1}-t_{2}\right\|<\delta}\left\|x\left(t_{1}\right)-x\left(t_{2}\right)\right\| .
$$

It was proved in [13] that $\Phi$ is well defined. (i.e., there is $E_{0} \in \Delta(\Omega)$ which achieves the maximum in (3.15)) and is a monotone, nonsingular and regular MNC.

Let us choose a constant $L>0$, such that

$$
p=\left(2 M_{1}+4 M_{1}^{2} M_{2}\left\|K_{W}\right\|_{L^{1}}\right) \sup _{t \in[0, b]} \int_{0}^{t} \eta(s) e^{-L(t-s)} d s<1,
$$

where $M_{1}=\sup \{\|U(t, s)\|:(t, s) \in J \times J\}$ and $\eta$ is the integrable function in the hypothesis $(H 2)$. Let $G_{y}=G_{1} y+G_{2} y$ as defined in theorem (3.1). From the regularity of $\Phi$, it is enough to prove that $\Phi(W)=(0,0)$. Since $\Phi(G(W))$ is a maximum, let $\left\{z^{(n)}\right\}_{n=1}^{\infty} \subseteq G(W)$ be the denumerable set which achieves its maximum. Then there exists a set $\left\{y^{(n)}\right\}_{n=1}^{\infty} \subseteq W$ such that

$$
z^{(n)}(t)=\left(G y^{(n)}\right)(t)=\left(G_{1} y^{(n)}\right)(t)+\left(G_{2} y^{(n)}\right)(t) \text {, for all } n \geq 1, t \in[0, b] .
$$

Now we give an estimation for $\alpha\left(\left\{z^{(n)}\right\}_{n=1}^{\infty}\right)$. Since $I_{i}(\cdot)$ is compact, we get

$$
\beta\left(\left\{\left(G_{1} y^{(n)}\right)(t)\right\}_{n=1}^{\infty}\right)=0, \text { for } t \in[0, b] .
$$

From $(3.12),(3.13)$, noticing that $K_{i}=0$, as $I_{i}$ is compact, we have that

$$
\begin{aligned}
& \beta\left(\left\{\left(G_{2} y^{(n)}\right)(t)\right\}_{n=1}^{\infty}\right) \\
& \leq 2 M_{1} \int_{0}^{t} \eta(s) \sup _{0 \leq \tau \leq s} \beta\left(\left\{y^{(n)}(\tau)\right\}_{n=1}^{\infty}\right) d s \\
& \quad+4 M_{1}^{2} M_{2}\left\|K_{W}\right\|_{L^{1}} \int_{0}^{t} \eta(s) \sup _{0 \leq \tau \leq s} \beta\left(\left\{y^{(n)}(\tau)\right\}_{n=1}^{\infty}\right) d s \\
& \quad\left(2 M_{1}+4 M_{1}^{2} M_{2}\left\|K_{W}\right\|_{L^{1}}\right) \int_{0}^{t} \eta(s) \sup _{0 \leq \tau \leq s} \beta\left(\left\{y^{(n)}(\tau)\right\}_{n=1}^{\infty}\right) d s \\
& \leq\left(2 M_{1}+4 M_{1}^{2} M_{2}\left\|K_{W}\right\|_{L^{1}}\right) \int_{0}^{t} \eta(s) e^{L s} \sup _{t \in[0, b]}\left(e^{-L t} \beta\left(\left\{y^{(n)}(t)\right\}_{n=1}^{\infty}\right)\right) d s \\
& =\left(2 M_{1}+4 M_{1}^{2} M_{2}\left\|K_{W}\right\|_{L^{1}}\right) \alpha\left(\left\{y^{(n)}\right\}_{n=1}^{\infty}\right) \int_{0}^{t} \eta(s) e^{L s} d s, \text { for } t \in[0, b] .
\end{aligned}
$$

From (3.18) and (3.19), it follows that

$$
\begin{aligned}
& \alpha\left(\left\{z^{(n)}\right\}_{n=1}^{\infty}\right)=\sup _{t \in[0, b]} e^{-L t} \beta\left(\left\{\left(G_{1} y^{(n)}\right)(t)+\left(G_{2} y^{(n)}\right)(t)\right\}_{n=1}^{\infty}\right) \\
\leq & \sup _{t \in[0, b]} e^{-L t}\left(2 M_{1}+4 M_{1}^{2} M_{2}\left\|K_{W}\right\|_{L^{1}}\right) \alpha\left(\left\{y^{(n)}\right\}_{n=1}^{\infty}\right) \int_{0}^{t} \eta(s) e^{L s} d s \\
= & \alpha\left(\left\{y^{(n)}\right\}_{n=1}^{\infty}\right)\left(2 M_{1}+4 M_{1}^{2} M_{2}\left\|K_{W}\right\|_{L^{1}}\right) \sup _{t \in[0, b]} \int_{0}^{t} \eta(s) e^{-L(t-s)} d s \\
= & \alpha\left(\left\{y^{(n)}\right\}_{n=1}^{\infty}\right) p .
\end{aligned}
$$


Therefore, we have that

$$
\alpha\left(\left\{y^{(n)}\right\}_{n=1}^{\infty}\right) \leq \alpha(W) \leq \alpha(\overline{c o}(\{0\} \cup G(W)))=\alpha\left(\left\{z^{(n)}\right\}_{n=1}^{\infty}\right) \leq \alpha\left(\left\{y^{(n)}\right\}_{n=1}^{\infty}\right) p .
$$

From (3.16), we obtain that

$$
\alpha\left(\left\{y^{(n)}\right\}_{n=1}^{\infty}\right)=\alpha(W)=\alpha\left(\left\{z^{(n)}\right\}_{n=1}^{\infty}\right)=0 .
$$

From the definition of $\alpha$, we have

$$
\beta\left(\left\{y^{(n)}(t)\right\}_{n=1}^{\infty}\right)=\beta\left(\left\{z^{(n)}(t)\right\}_{n=1}^{\infty}\right)=0, \text { for every } t \in[0, b] .
$$

From (3.12) and (3.20), noticing that $K_{i}=0$ in (3.12), we get that

$$
\begin{aligned}
& \beta\left(\left\{f\left(t, y_{t}^{(n)}+\hat{\varphi}_{t}\right)+\left(B u_{y^{(n)}}\right)(t)\right\}_{n=1}^{\infty}\right) \\
& \leq \eta(t) \sup _{-r \leq \theta \leq 0} \beta\left(\left\{y^{(n)}(t+\theta)+\hat{\varphi}(t+\theta)\right\}_{n=1}^{\infty}\right) \\
& \quad+2 M_{1} M_{2} K_{W}(s) \int_{0}^{b} \eta(s) \sup _{0 \leq \tau \leq s} \beta\left(\left\{y^{(n)}(\tau)\right\}_{n=1}^{\infty}\right) d s \\
& \leq \eta(t) \sup _{0 \leq \tau \leq t} \beta\left(\left\{y^{(n)}(\tau)\right\}_{n=1}^{\infty}\right) \\
& \quad+2 M_{1} M_{2} K_{W}(s) \int_{0}^{b} \eta(s) \sup _{0 \leq \tau \leq s} \beta\left(\left\{y_{n}(\tau)\right\}_{n=1}^{\infty}\right) d s=0,
\end{aligned}
$$

That is, $\left\{f\left(t, y_{t}^{(n)}+\hat{\varphi}_{t}\right)+B u_{y^{(n)}}(t)\right\}_{n=1}^{\infty}$ is relatively compact for almost all $t \in[0, b]$ in $X$. Moreover, from the fact that $\left\{y^{(n)}\right\}_{n=1}^{\infty} \subseteq B_{q}$, by $(H 2)(i i)$ and (3.6), it is easy to see that $\left\{f\left(t, y_{t}^{(n)}+\hat{\varphi}_{t}\right)+B u_{y^{(n)}}(t)\right\}_{n=1}^{\infty}$ is uniformly integrable for a.e. $t \in[0, b]$. So $\left\{f\left(\cdot, y^{(n)}+\hat{\varphi}\right)+B u_{y^{(n)}}\right\}_{n=1}^{\infty}$ is semicompact according to the Definition 2.5. By applying Lemma 2.4, we have that $G_{2}\left(\left\{y^{(n)}\right\}_{n=1}^{\infty}\right)$ is relatively compact in $\mathcal{P C}_{0}$.

On the other hand, by the strong continuity of $U(t, s)$ and the compactness of $I_{i}$, we can easily verify that $G_{1}\left(\left\{y^{(n)}\right\}_{n=1}^{\infty}\right)$ is relatively compact. Then by $(3.17),\left\{z^{(n)}\right\}_{n=1}^{\infty}$ is also relatively compact in $\mathcal{P} \mathcal{C}_{0}$. Since $\Phi$ is a monotone, nonsingular, regular MNC, from Monch's condition, we have that

$$
\Phi(W) \leq \Phi(\overline{c o}(\{0\} \cup G(W)))=\Phi\left(\left\{z_{n}\right\}_{n=1}^{\infty}\right)=(0,0) .
$$

Therefore, $W$ is relatively compact in $\mathcal{P C}_{0}$. This completes the the proof.

\section{Example}

In this section, we give an example to illustrate our results above.

Example 4.1. Consider the impulsive partial system of the form

$$
\begin{aligned}
\frac{\partial}{\partial t} z(t, \xi) & =\frac{\partial}{\partial \xi} z(t, \xi)+m(\xi) u(t, \xi)+F(t, z(t-r, \xi)) \\
\text { for } \xi \in[0, \pi], t \in[0, b], t \neq t_{i}, i=1,2, \ldots, s & \\
z\left(t_{i}^{+}, \xi\right) & -z\left(t_{i}^{-}, \xi\right)=I_{i}\left(z\left(t_{i}^{-}, \xi\right)\right), \xi \in(0, \pi], i=1,2, \ldots, s \\
z(t, 0) & =z(t, \pi)=0, t \in[0, b], \\
z(t, \xi) & =\varphi(t, \xi), t \in[-r, 0], \xi \in[0, \pi],
\end{aligned}
$$

where $r>0, I_{i}>0, i=1,2, \ldots, s, \varphi \in \mathcal{D}=\{\psi:[-r, b] \times[0, \pi] \rightarrow \mathbb{R} ; \psi$ is continuous everywhere except for a countable number of points at which $\psi\left(s^{-}\right), \psi\left(s^{+}\right)$exists with $\left.\psi\left(s^{-}\right)=\psi(s)\right\}, 0=t_{0}<t_{1}<t_{2}<\cdots<$ $t_{s+1}=b, z\left(t_{i}^{+}\right)=\lim _{(h, \xi) \rightarrow\left(0^{+}, \xi\right)} z\left(t_{i}+h, \xi\right), z\left(t_{i}^{-}\right)=\lim _{(h, \xi) \rightarrow\left(0^{-}, \xi\right)} z\left(t_{i}+h, \xi\right), F:[0, b] \times \mathbb{R} \rightarrow \mathbb{R}, B: X \rightarrow X$. 
Let

$$
\begin{aligned}
& x(t)(\xi)=z(t, \xi), t \in[0, b], \xi \in[0, \pi], \\
& I_{i}\left(x\left(t_{i}^{-}\right)\right)(\xi)=I_{i} z\left(t_{i}^{-}, \xi\right), \xi \in[0, \pi], i=i, 2, \ldots, s, \\
& F(t, \varphi)(s)=F(t, \varphi(\theta, \xi)), \theta \in[-r, 0], \xi \in[0, \pi], \\
& \varphi(\theta)(\xi)=\varphi(\theta, \xi), \theta \in[-r, 0], \xi \in[0, \pi], \\
& (B u)(\xi)=m(\xi) u(\xi), \xi \in[0, \pi] .
\end{aligned}
$$

Take $X=L^{2}[0, \pi]$ and define $A(t) \equiv A: D(A) \subset X \rightarrow X$ by $A w=w^{\prime}$ with domain $D(A)=\left\{w \in X: w^{\prime} \in\right.$ $X, w(\xi)=w(0)=0\}$. It is well known that $A$ is an infinitesimal generator of a semigroup $T(t)$ defined by $T(t) w(s)=w(t+s)$ for each $w \in X$. T(t) is not a compact semigroup on $X$ and $\beta(T(t) D) \leq \beta(D)$, where $\beta$ is the Hausdorff MNC.

Then, the system $(4.1)-(4.4)$ is the abstract formulation of the system $(1.1)-(1.3)$. We can conclude that the system $(4.1)-(4.4)$ is controllable on $[0, \mathrm{~b}]$.

\section{Acknowledgements}

The first author thanks to Mr. Muthuvel Ramaswamy, Secretary, Muthayammal College of Arts and Science, Rasipuram- 637 408, Tamil Nadu, India, for his constant encouragements and support for this research work.

The second author dedicates this paper to Silver Jubilee Year Celebrations of Karunya University, Coimbatore-641 114, Tamil Nadu, India. And also the authors wish to thank Dr. Paul Dhinakaran, Chancellor, Dr. Paul P. Appasamy, ViceChancellor, and Dr(Mrs). Anne Mary Fernandez, Registrar, of Karunya University, Coimbatore, for their constant encouragements and support for this research work.

\section{References}

[1] A. Anguraj and M. Mallika Arjunan, Existence and uniqueness of mild and classical solutions of impulsive evolution equations, Electron J. Differential Equations, 111 (2005), 1-8. 1

[2] A. Anguraj and M. Mallika Arjunan, Existence results for an impulsive neutral integro-differential equations in Banach spaces, Nonlinear Stud., 16(1)(2009), 33-48. 1

[3] D.D. Bainov and P.S. Simeonov, Impulsive Differential Equations: Periodic Solutions and Applications, Longman Scientific and Technical Group, England, 1993. 1

[4] J. Banas and K. Goebel, Measure of Noncompactness in Banach Spaces, in: Lecture Notes in Pure and Applied Matyenath, Marcel Dekker, New York, 1980. 2.1 2.1

[5] M. Benchohra, J. Henderson and S.K. Ntouyas, Existence results for impulsive multivalued semilinear neutral functional inclusions in Banach spaces, J. Math. Anal. Appl., 263(2001), 763-780. 1

[6] L. Chen and G. Li, Approximate controllability of impulsive differential equations with nonlocal conditions, Int. J. Nonlinear Sci., 10(2010), 438-446. 1

[7] Z. Fan, Impulsive problems for semilinear differential equations with nonlocal conditions, Nonlinear Anal., 72(2010), 1104-1109. 1

[8] Z. Fan and G. Li, Existence results for semilinear differential equations with nonlocal and impulsive conditions, J. Funct. Anal., (258)(2010), 1709-1727. 1

[9] E. Hernandez, M. Pierri and G. Goncalves, Existence results for an impulsive abstract partial differential equation with state-dependent delay, Comput. Math. Appl., 52(2006), 411-420. 1

[10] E. Hernandez M., M. Rabello and H. Henriaquez, Existence of solutions for impulsive partial neutral functional differential equations, J. Math. Anal. Appl., 331(2007), 1135-1158. 1

[11] S. Ji, G. Li and M. Wang, Controllability of impulsive differential systems with nonlocal conditions, Appl.Math. Comput., 217(2011), 6981-6989. 1 ]

[12] S. Ji and S. Wen, Nonlocal cauchy problem for Impulsive differential equations in Banach spaces, Int. J. Nonlinear Sci., 10(2010), 88-95. 1

[13] M. Kamenskii, V. Obukhovskii and P. Zecca, Condensing Multivalued Maps and Semilinear Differential Inclusions in Banach Spaces, De Gruyter, 2001. 2.1, 3, 3

[14] V. Lakshmikantham, D.D. Bainov and P.S. Simeonov, Theory of Impulsive Differential Equations, World Scientific, Singapore, 1989. 1 
[15] M. Li, M. Wang and F. Zhang, Controllability of impulsive functional differential systems in Banach spaces, Chaos, Solitons and Fractals, 29(2006), 175-181. 1

[16] H. Monch, Boundary value problems for nonlinear ordinary differential equations of second order in Banach spaces, Nonlinear Anal., 4(1980), 985-999. 2.5

[17] V. Obukhovski and P. Zecca, Controllability for systems governed by semilinear differential inclusions in a Banach space with a noncompact semigroup, Nonlinear Anal., 70(2009), 3424-3436. 1. 2.3, 2.4

[18] A. Pazy, Semigroups of Linear Operators and Applications to Partial Differential Equations, Springer-Verlag, Newyork, 1983. 2

[19] B. Radhakrishnan and K. Balachandran, Controllability of impulsive neutral functional evolution integrodifferential systems with infinite delay, Nonlinear Anal., 5(2011), 655-670. 1

[20] A.M. Samoilenko and N.A. Perestyuk, Impulsive Differential Equations, World Scientific, Singapore, 1995.1

[21] C. Travis and G. Webb, Existence and stability for partial functional differential equations, Trans. Amer. Math. Soc., 200(1974), 395-418. 1

[22] G.Webb, An abstract semilinear Volterra integrodifferential equations, Proc. Amer. Math. Soc., 69(1978), 255260. 1

[23] R. Ye, Existence of solutions for impulsive partial neutral functional differential equation with infinite delay, Nonlinear Anal., 73(2010), 155-162. 1.2 .2 